CORRECTION

https://doi.org/10.1038/s41586-018-0229-5

\title{
Author Correction: Inflammatory memory sensitizes skin epithelial stem cells to tissue damage
}

Shruti Naik, Samantha B. Larsen, Nicholas C. Gomez, Kirill Alaverdyan, Ataman Sendoel, Shaopeng Yuan,

Lisa Polak, Anita Kulukian, Sophia Chai \& Elaine Fuchs

Correction to: Nature https://doi.org/10.1038/nature24271, published online 18 October 2017.

In Fig. $2 \mathrm{~g}$ of this Article, a panel was inadvertently duplicated. The 'D30 IMQ' image was a duplicate of the 'D6 Ctrl' image. Figure $2 \mathrm{~g}$ has been corrected online to show the correct 'D30 IMQ' image (showing skin inflammation induced by the NALP3 agonist imiquimod, IMQ). The Supplementary Information to this Amendment contains the old, incorrect Fig. 2 for transparency.

Supplementary information is available for this Amendment at https://doi.org/ 10.1038/s41586-018-0229-5. 